\title{
Critical Limb Ischemia In A Diabetes Mellitus Patient with Atrial Fibrilation
}

\author{
Caesar Lagaliggo Givani ${ }^{1,}$ Hermina Novida ${ }^{2 *}$ \\ ${ }^{1}$ Department of Internal Medicine, Faculty of Medicine, Universitas Airlangga - Dr. Soetomo General Hospital Surabaya, \\ Indonesia. \\ ${ }^{2}$ Division of Endocrinology and Metabolic Disease, Department of Internal Medicine, Faculty of Medicine, Universitas \\ Airlangga - Dr. Soetomo General Hospital Surabaya, Indonesia.
}

\begin{abstract}
A B S T R A C T
Critical limb ischemia (CLI) is a clinical syndrome in the form of ischemic pain, especially at rest or a tissue loss condition, such as an ulcer or gangrene that does not heal, associated with peripheral arterial disease (PAD). Diabetes mellitus (DM) accelerates atherosclerosis and becomes one of the risks of PAD. It is also known to accelerate the worsening of PAD with a $4 \mathrm{x}$ greater risk of developing CLI compared to patients without DM. At the other side, $60-95 \%$ of patients who are operated on as a result of limb ischemia are diagnosed with atrial fibrillation (AF). This paper is a case report regarding a patient with CLI as a complication of DM and AF.
\end{abstract}

Keywords: Critical limb ischemia, peripheral arterial disease, diabetes mellitus, atrial fibrillation

Correspondence: Hermina Novida

E-mail: hermina-n@ffk.unair.ac.id

Article history: • Received 19 August $2020 \bullet$ Received in revised form 28 August 2020• Accepted 31 August 2020

\section{INTRODUCTION}

Critical limb ischemia (CLI) is a clinical syndrome in the form of ischemic pain, especially at rest or a tissue loss condition, such as an ulcer or gangrene that does not heal, associated with peripheral arterial disease (PAD) (Kinlay, 2016). CLI is the most severe spectrum of PAD which is often characterized by progressive claudication that may develop to nocturnal rest pain (Beard, 2000; Kinlay, 2016; Spreen, et al., 2016). CLI prevalence in the United States is around $12 \%$, and $20 \%$ of those affected are over 70 years old. CLI is more common in men than women, and several factors such as smoking and a history of diabetes increase the risk of developing CLI (Bitar and Garcia, 2010).

Diabetes mellitus (DM) accelerates atherosclerosis and becomes one of the risks of PAD ( $\mathrm{Li}$ et al., 2007). Additionally, DM is also known to accelerate the worsening of PAD with a $4 \mathrm{x}$ greater risk of developing CLI compared to patients without DM (Spreen et al., 2016). Peripheral neuropathy that often accompanies PAD incidence in DM often causes the diagnosis to be under-recognized so that it is hypothesized as one of the factors that develop PAD into CLI (Potier et al., 2011, Thiruvoipati et al., 2015, Spreen et al., 2016).

Although often overlooked, atrial fibrillation (AF) must be considered as the most common cause of embolism in peripheral arteries. $60-95 \%$ of patients who are operated on as a result of limb ischemia are diagnosed with AF. Thromboembolism caused by AF is often found in women (5.7 times) than men ( 4 times) when compared to patients without heart rhythm abnormalities. The higher prevalence in these women is thought to be related to differences in the coagulation process and endothelial function (Wasilewska and Gosk-Bierska, 2013).
$\mathrm{AF}$ is the most common heart rhythm abnormality, around $1-2 \%$ in the general population. This event increases with age (AHA, 2011, Kuzniatsova and Lip, 2010). Thromboembolism is the most severe complication of AF. Early diagnosis and appropriate treatment can improve the prognosis of systemic thromboembolism due to AF (Menke et al., 2010). This paper is a case report regarding a patient with CLI as a complication of DM and AF.

\section{CASE REPORT}

26th June 2019

A Woman 48 years old admitted by his family to the outpatient clinic of Dr. Soetomo General Hospital, Surabaya, with a chief complaint of pain in the left foot.

\section{Current medical history}

The patient complained about having pain in both right and left feet since 2 months ago (the pain was worse in the left foot). The pain was felt especially during activity and disappeared after rest. The location of the pain was felt from the groin to the tip of the left toe. The pain was aggravated by activity and improved with rest. Last month, she felt that the pain was increasingly more severe and continued even at rest-the complaints were accompanied by tingling and numbness.

The patient also complained about having a left leg wound since last month. Her left toes began to turn black. She denied any previous fever and any history of trauma.

The patient often had heart palpitation, weakness, and fatigue since 3 months ago. She experienced no shortness of breath and no chest pain. 


\section{Previous medical history}

The patient was diagnosed with type-2 DM 3 years ago, had been prescribed metformin $3 \times 500 \mathrm{mg}$ during initial diagnosis. She discontinued the medication by herself. She has no history of hypertension.

\section{Familial medical history}

There is no family member that experiences similar symptoms. The patient's mother also has DM.

\section{Socioeconomic history}

The patient works as a farmer in Pare and as a housewife. The patient is married and has 2 children aged $21 \& 16$ years old. Her husband also works as a farmer.

\section{Physical examination}

From vital signs examination, we obtained a general state of weakness with GCS 456. Blood pressure 100/60 $\mathrm{mmHg}$, pulse $130 \mathrm{x} /$ minute, irregular rhythm, normal amplitude. Respiratory rate of 20x/minute with $97 \% \quad \mathrm{SpO}_{2}$ with free oxygen. Axillary temperature of $36.8^{\circ} \mathrm{C}$. Pain scale assessment with Visual Analog Scale (VAS) obtained a score of 5 (moderate pain). The patient's body weight was $50 \mathrm{~kg}$, body height was $150 \mathrm{~cm}$, with a body mass index of $22 \mathrm{~kg} / \mathrm{m} 2$ (normal).

From the head and neck examination, we obtained anemic conjunctiva, non-icteric sclera, no cyanosis, no dyspnea. There was no increase in jugular venous pressure nor any enlargement of the neck lymph nodes. There were no stomatitis nor oral candidiasis.

From the chest examination, we obtained symmetrical chest wall movement, without intercostal or supraclavicular retraction. On cardiac examination, irregular S1 and S2 were obtained, diastolic murmurs were found in apex. In lung examination, vesicular breath sounds were obtained on both hemithorax, no additional breath sounds, either rhonchi or wheezing, in both lung fields.

From abdominal examination, we obtained a flat abdomen with normal bowel sounds, tympanic percussion, and supple on palpation. No tenderness was obtained, with the liver and the spleen were not palpable.

From extremities examination, it felt warm, dry, and looked red. No edema was seen in both extremities. There was a necrotic tissue in the $1^{\text {st }}$ and $2^{\text {nd }}$ digiti of the left inferior extremity.

Table 1. Extremities examination, it felt warm, dry, and looked red

\begin{tabular}{ccc}
\hline Arterial pulsation & Right & Left \\
\hline Femoral artery & ++ & + \\
\hline Poplitea artery & ++ & + \\
\hline Posterior tibial artery & ++ & - \\
\hline Dorsalis pedis artery & ++ & - \\
\hline
\end{tabular}

Table 2. Periphery oxygen saturation examination of each toe

\begin{tabular}{ccc}
\hline Oxygen saturation & Right & Left \\
\hline Digiti I & $99 \%$ & $83 \%$ \\
\hline Digiti II & $99 \%$ & $84 \%$ \\
\hline Digiti III & $99 \%$ & $92 \%$ \\
\hline Digiti IV & $99 \%$ & $92 \%$ \\
\hline Digiti V & $99 \%$ & $94 \%$ \\
\hline
\end{tabular}

\section{Additional examinations}

From laboratory examinations $(26 / 06 / 19)$ we found $\mathrm{Hb}$ 9.0 g/dL, Hct 25.7\%, Leucocytes 12.060/uL, Neutrophil $76 \%$, Lympocyte $15.1 \%$, Platelets 358.000 . random blood sugar $254 \mathrm{mg} / \mathrm{dL}$, BUN $10 \mathrm{mg} / \mathrm{dL}$, creatinine $0.9 \mathrm{mg} / \mathrm{dL}$, SGOT $46 \mathrm{u} / \mathrm{L}$, SGPT $31 \mathrm{u} / \mathrm{L}$, Albumin $4.0 \mathrm{~g} / \mathrm{dl}$, Natrium $135 \mathrm{mmol} / \mathrm{l}$, Potassium $4.1 \mathrm{mmol} / \mathrm{L}$, Chloride $96 \mathrm{mmol} / \mathrm{L}$, and a non-reactive HbsAg and anti-HCV examination.

From radiology examination of AP chest Xray we found cardiomegaly with 70\% CTR. From electrocardiography $(26 / 06 / 19)$ we found atrial fibrillation with ventricular response of 100-150x/minute, normal frontal axis, counterclockwise horizontal axis.

From ABI (Ankle Brachial Index) examination we found Right $\mathrm{ABI}$ : 0.62 dan Left $\mathrm{ABI}$ 0.55. It may indicates mild to moderate peripheral arterial diseases (PAD).

From lower extrimities USG Duplex examination, $(16 / 05 / 2019)$ There are significant stenosis of left external iliac artery, occlusion from external iliac artery to communicating femoral artery of left leg, and significant stenosis of communicating femoral artery of right leg.

From the CT angiography of left leg (17/05/2019) there are total occlusion of left external iliac artery, left popliteal artery (1/3 mid to distal), left anterior tibial artery, right profound femoral artery, right communicating femoral artery, right anterior tibial artery, and right posterior tibial artery. Moderate stenosis of left communicating iliac artery to branches of left external iliac artery, left profound femoral artery (1/3 mid to lower), left posterior tibial artery. Mild stenosis of right anterior tibial artery (1/3 upper) and posterior tibial artery (1/3 upper).

From echocardiography examination (16/5/19), we found normal $\mathrm{RV}$, dilated LA dan RA, hyperthropic concentric LV, with normal LV systolic function (EF 65.5\% Biplane). Abnormal diastolic LV function, abnormal systolic RV function, hypokinecity of anteroseptal-anterioranterolateral at the height of (B-M). Moderate MS, mild $\mathrm{MR}$, moderate AR, mild PR, mild TR, minimal pericardial efussion without any chamber collapse, EC (+) in LA, PH intermediate probability.

\section{Assestment and management}

Based on the history, physical and auxiliary examinations, the patient's assessment is critical limb ischemia of both lower extremities, necrosis of left foot digiti I, atrial fibrillation, moderate mitral stenosis, moderate mitral regurgitation, anemia, and type 2 Diabetes Mellitus.

Patient is planned for hospitalization. Patient is also planned for arteriography and percutaneous transluminal angiography (PTA). Therapy given to the patient is of $\mathrm{NaCl}$ $0.9 \%$ infusion $500 \mathrm{cc}$ for 24 hours, Acetylsalicylic acid 100 mg every 24 hours, Atorvastatin $40 \mathrm{mg}$

every 24 hours, Beraprost sodium 20 mcg every 8 hours, Cilostazol 50 mg every 12 hours, Bisoprolol 5 mg every 24 hours. Warfarin is paused.

\section{Diasease Course}

27th June 2019

Result of Arteriography and Percutaneous Transuminal Angioplasty (PTA) of the left lower extrimity using local anesthesia:

- External iliac artery: non-significant stenosis 30\%

- Femoral artery: Total occusion from CFA to deep femoral artery (thrombus is shown). 
- Poplitea artery: Normal

- Anterior tibial artery: Normal

- Posterior tibial artery: Normal

- Peroneal artery: Diffuse disease

After the arteriography and PTA, the patient's general condition was good, no complaints of tightness, chest pain, or palpitations. She experienced pain on the punctured area. From physical examination, we obtained GCS 456; BP: 99/67; HR: 90x / min; RR: 18x / min; T: 36.5 .

The patient is planned for 2 bags of PRC transfusion, additional complete blood count, hemostatic function, and INR. She is also planned to have her HbA1C, fasting blood glucose, 2- hours postprandial blood glucose, TSHs, and free T4 checked. Continued previous therapy with warfarin $2 \mathrm{mg}$ every 24 hours may be resume. Paracetamol $500 \mathrm{mg}$ every 8 hours if needed.

Table 3. Vascular status examination of Department of Thoracic, Cardiac, and Vascular Surgery. (28 $8^{\text {th }}$ July 2019)

\begin{tabular}{lll}
\hline $\begin{array}{c}\text { Arterial } \\
\text { pulsation }\end{array}$ & \multicolumn{1}{c}{ Right } & \multicolumn{1}{c}{ Left } \\
\cline { 2 - 3 } & $\begin{array}{c}\text { Palpation/ } \\
\text { doppler }\end{array}$ & $\begin{array}{c}\text { Palpation/ } \\
\text { Doppler }\end{array}$ \\
\hline Femoral artery & $++/+$ & $++/+$ \\
\hline Popliteal artery & $++/+$ & $++/+$ \\
\hline Posterior tibial artery & $++/+$ & $++/+$ \\
\hline Dorsalis pedis artery & $++/+$ & $++/+$ \\
\hline $\begin{array}{l}\text { *Warm compress and necrotomy of left foot digiti 1 were } \\
\text { performed }\end{array}$
\end{tabular}

Table 4. Pain in the digiti I left toe after necrotomy.

\begin{tabular}{lll}
\hline \multicolumn{1}{c}{$\begin{array}{c}\text { Arterial } \\
\text { pulsation }\end{array}$} & Right & Left \\
\hline Femoral artery & +++ & ++ \\
\hline Popliteal artery & +++ & ++ \\
\hline $\begin{array}{l}\text { Posterior tibial } \\
\text { artery }\end{array}$ & +++ & ++ \\
\hline $\begin{array}{l}\text { Dorsalis pedis } \\
\text { artery }\end{array}$ & +++ & ++ \\
\hline *BP: $100 / 60 ; \mathrm{HR}: 92 \mathrm{x} / \mathrm{m} ; \mathrm{RR}: 18 \mathrm{x} / \mathrm{m} ; \mathrm{T}: 36.7^{\circ} \mathrm{C}$
\end{tabular}

Table 5. Examination of peripheral oxygen saturation

\begin{tabular}{lcc}
\hline Saturation & Right & Left \\
\hline Digiti I & $99 \%$ & $\begin{array}{c}\text { Covered in gauze after } \\
\text { necrotomy }\end{array}$ \\
\hline Digiti II & $99 \%$ & $98 \%$ \\
\hline Digiti III & $99 \%$ & $98 \%$ \\
\hline Digiti IV & $99 \%$ & $98 \%$ \\
\hline Digiti V & $99 \%$ & $98 \%$ \\
\hline
\end{tabular}

*Blood laboratory evaluation results: Hb 10; WBC 6,500; Plt 425,000; fasting blood glucose 140; 2-hours pp blood glucose 231. A1C: 7

2nd July 2019

The patient was discharged and advised to control regularly in cardiovascular, cardiovascular-thoracic Surgery, and internal medicine (endocrine) clinics. The final diagnosis was CLI of the inferior extremities post digiti I left foot necrotomy \& post left inferior extremity PTA, necrosis of the digiti I left foot post necrotomy, AF, moderate mitral stenosis, moderate mitral regurgitation, anemia, and type 2 DM.

Outpatient therapy: Acetylsalicylic acid $100 \mathrm{mg}$ every 24 hours, Warfarin $3 \mathrm{mg}$ every 24 hours, Atorvastatin $40 \mathrm{mg}$ every 24 hours, Bisoprolol $5 \mathrm{mg}$ every 12 hours, paracetamol $500 \mathrm{mg}$ every 8 hours, Sulfas Ferrous every 8 hours, glimepiride $2 \mathrm{mg}$ every 24 hours, and metformin $500 \mathrm{mg}$ every 8 hours. Conservative treatment for mitral Stenosis and mitral regurgitation. Further clinical evaluation from polyclinic.

\section{DISCUSSION}

CLI is quite dangerous as a high-risk factor for limb loss and increases cardiovascular events.(Davies, 2012, Kinlay, 2016) $25 \%$ of patients with CLI die within one year, and more than $60 \%$ of patients are estimated to die within five years (Davies, 2012). CLI is a leading cause of amputation in the United States; therefore, patients with CLI should be referred immediately to vascular surgeons for diagnostic and revascularization therapy with the primary goal of limb preservation (Beard, 2000, Nehler et al., 2014, Kinlay, 2016). Age is also a factor that plays a role in CLI occurrence, where the highest prevalence at $29 \%$ is found at the age above 70 years old, and at the age of 50-69 years old if accompanied by a 10-year history of diabetes or smoking (Davies, 2012).

CLI is the most severe spectrum of PAD, which is often characterized by progressive claudication that may develop to nocturnal rest pain (Beard, 2000, Kinlay, 2016). The stage of CLI can be divided into asymptomatic, claudication, CLI with rest pain, and CLI with tissue loss (Kinlay, 2016). In its early stages, the artery stenosis causes a decrease in blood flow to the lower extremities especially during activity, so the initial symptoms are claudication where the pain is more severe with activity and improves with rest. Along with the development of arteries narrowing, blood flow to the extremities is significantly reduced even while at rest which is called nocturnal rest pain (Hernando and Conejero, 2007). The pain often feels more severe at night when the patient is lying down, causing the patient to wake up from sleep, associated with reduced blood flow due to gravity. Patients often sleep with legs dangling or sleep while leaning to increase blood flow to the lower extremities so the pain is reduced (Kinlay, 2016).

The diagnosis of CLI is not only based on clinical symptoms but also requires examinations to assess the disturbed blood flow. Typical physical examination includes loss of palpation of the dorsal pedis artery, shiny leg skin, and calf muscle wasting. The diagnostic criteria for CLI

according to Santilli (1999) are pain complaints in the lower extremities during rest for more than two weeks that require regular use of analgesics; or sores that do not heal within 4-12 weeks with optimal therapy or the presence of gangrene (tissue necrosis) in the feet or toes, especially in the distal area; accompanied systolic blood pressure examination at the ankle $\leq 50 \mathrm{mmHg}$ or on the digiti I foot $\leq 30 \mathrm{mmHg}$; while Kinlay (2016) states ischemia as hemodynamic criteria for systolic pressure at ankle $\leq 40$ $70 \mathrm{mmHg}$, digiti I foot $\leq 30-50 \mathrm{mmHg}$, and transcutaneous oxygen $(\mathrm{TcO} 2) \leq 20-40 \mathrm{mmHg}$.

In addition to history-taking and physical examination that leads to ischemia, other objective examinations are also needed, such as measuring blood flow with a hand- 
held Doppler, measuring ankle pressure, and ABI (Santilli, 1999; Davies, 2012, Kinlay, 2016). A low ABI number is a predictor of increased mortality from CLI (Davies, 2012). Duplex ultrasound (DUS) examination, non-invasive angiographies such as CTA (computerized tomography) or MRA (magnetic resonance) can show blood vessels obstructions. CTA and MRA can help to localize targets and to assist in determining revascularization. Blood vessels mapping is also necessary if an autologous venous surgical bypass is planned (Kinlay, 2016).

From history-taking, we obtained complaints of pain in both feet (left foot was worse) that she had felt for two months. She initially experienced pain, especially during activity and improved with rest, but starting from last month, she continuously felt the pain and did not improve even when resting. In VAS examination, a value of 5 was obtained, meaning moderate pain. The complaints were also accompanied by a tingling sensation and numbness. In addition to pain, a wound that has not healed since one month ago was also present; the toes also began to turn black. On physical examination, necrotic tissue was seen in digiti I and II of left lower extremity. During arterial pulsations palpation, there was a decrease in pulsation in the popliteal arteries and non-palpable posterior tibial artery pulses and dorsal pedis arteries in both of her lower extremities. We also obtained oxygen saturation reduction in digiti I and II of left lower extremity.

Doppler ultrasound examination was performed on her lower limb, and significant stenosis was found in left external iliac artery to communicating femoral artery of left leg, and in communicating femoral artery of right leg. CT Scan Angiography (CTA) examination revealed a total occlusion of left external iliac artery, left popliteal artery (1/3 mid to distal), left anterior tibial artery, right profound femoral artery, right communicating femoral artery, right anterior tibial artery, and right posterior tibial artery. From history-taking, physical examination, and auxiliary examinations, the patient was diagnosed with pedis region CLI of both lower extremities.

Chronic complications of DM are divided into macrovascular and microvascular. Macrovascular complications include cardiovascular disease, stroke, and PAD (Papatheodorou, et al., 2018). DM increases the risk of PAD events by 2.72 times.(Thiruvoipati et al., 2015) PAD prevalence in patients with DM ranging from 9.5$13.6 \%$, which is far higher than the general

population of $4 \%$ ( $\mathrm{Li}$, et al., 2007). Although the incidence rate is quite large, PAD is often under- recognized due to peripheral neuropathy complications that disguises complaints from PAD (Potier et al., 2011, Thiruvoipati et al., 2015).

Hyperglycemia, dyslipidemia, and insulin resistance in DM patients cause endothelial inflammation and dysfunction, blood cells abnormalities, and hemostasis disorders. This also causes changes in the walls of blood vessels. Endothelial blood vessels under normal circumstances will balance thrombosis and fibrinolysis to prevent plaque. In addition, the endothelium will also produce nitric oxide (NO) which works as a vasodilator, inhibits platelet activation, and reduces inflammation. In patients with DM, insulin resistance inhibits the production of NO (Thiruvoipati et al., 2015).

Oxidative stress caused by hyperglycemic conditions inhibits NO production. Other than that, oxidative stress causes inflammation which also increases procoagulant factors that support the thrombosis process.
Hypercoagulable state in patients with DM increases coagulant factors and decreases anticoagulant levels, accompanied by hemostatic disorders that cause platelet dysfunction leading to increased platelet aggregation. All of the conditions above eventually lead to thrombosis (Thiruvoipati et al., 2015).

The prognosis of PAD patients with DM depends on comorbidity, the presence of infection, neuropathy, and immunological status of the patient (Thiruvoipati et al., 2015). DM can accelerate atherosclerosis process and has a higher amputation risk of 5-10x higher because of its tendency in the distal arteries, as well as masking due to neuropathy and a higher risk of infection (Hernando and Conejero, 2007, Li et al., 2007). The therapy includes lifestyle changes, increased physical activity, low cholesterol and fat diet can improve glucose intolerance and cardiovascular status (Thiruvoipati et al., 2015) . (According to the recommendations of the American Diabetes Association, the expected target of blood sugar level is HbA1c less than 7\% (Hirsch et al., 2006). Optimal treatment also needs to be given to wounds that do not heal, such as antibiotics usage if signs of infection and debridement needs are obtained. Using the right shoe size while paying special attention to feet's hygiene and humidity can reduce the risk of feet trauma (Hirsch et al., 2006).

The patient was diagnosed with DM since three years ago. The patient received oral anti-diabetes drug, metformin, but did not routinely control or took the medication. There were no symptoms of DM triads of polyuria, polyphagia, and weight loss. From auxiliary examinations, we obtained her random blood glucose levels of $254 \mathrm{mg} / \mathrm{dl}$. She was then planned for fasting blood glucose, 2-hours pp blood glucose, and $\mathrm{HbA} 1 \mathrm{C}$ examinations. From the examination results, we obtained fasting blood glucose $140 \mathrm{mg} / \mathrm{dl}$, 2-hours pp blood glucose $231 \mathrm{mg} / \mathrm{dl}$, and 7\% $\mathrm{HbA} 1 \mathrm{C}$; therefore, we diagnosed she had type 2 DM. To control blood glucose levels, she got oral anti-diabetic medication consisted of glimepiride and metformin. Necrotic wounds were regularly treated with wound-care accompanied by broad-spectrum cefixime antibiotics.

Although often overlooked, AF must be considered the most common cause of embolism in peripheral arteries. In $60-95 \%$ of patients who are operated on due to limb ischemia are diagnosed with AF. Thrombus formation in AF patients is caused by atrial blood stasis, accompanied by endothelial dysfunction and hypercoagulability. Increased fibrinogen and D- dimer fibrin were also found in AF patients, both persistent and paroxysmal AF, thereby facilitating coagulation (Shariff, et al., 2012). Thromboembolism caused by AF is often more prevalent in women (5.7 times) than men (4 times) when compared to patients without heart rhythm abnormalities. Women with AF are thought to have differences in coagulation processes and endothelial function.(Wasilewska and GoskBierska, 2013) Coagulation factors such as von Willebrand factor, prothrombin fragment, and tissue plasminogen activator antigen also increase in the atrial endothelium of female patients with AF (Wasilewska and Gosk-Bierska, 2013).

Cardiogenic emboli are most commonly found in the extremity arteries, especially the inferior extremity. AF is the dominant risk factor for left atrium embolism, followed by myocardial infarction, endocarditis, heart valve disorders, atrial myxoma, valve prosthesis, unstable atherosclerosis plaque, cardioversion, and so on. $76 \%$ of patients with 
peripheral embolism have cardiac comorbidities, with an AF prevalence of 55\% (Wasilewska and Gosk-Bierska, 2013).

$\mathrm{AF}$ is the most common heart rhythm abnormality, with around $1-2 \%$ in the general population. The prevalence of this condition increases with age (AHA, 2011, Kuzniatsova and Lip, 2010). Treatment of underlying disease and immediate risk factors can prevent not only AF but also improve prognosis.(Brandes et al., 2018) Rate control is preferred over rhythm control in most patients with AF. The treatment options are non-dihydropyridine CCB and betablockers with a pulse target of $<100 \mathrm{x} / \mathrm{min}$. Anticoagulants usage is recommended for ischemic stroke prevention by also considering the risk of bleeding.

From the physical examination, there were irregular heart sound and diastolic murmur on the apex. Electrocardiography examination revealed $\mathrm{AF}$ and occasional PVC. On echocardiography, the SEC is found on the left atrium, which indicated stasis, leading to the risk of thromboembolism. Cardiogenic thromboembolism was most common in the inferior limb. The patient got betablockers (bisoprolol) and digoxin as rate controllers. The use of antiplatelet (aspirin) and anticoagulants (warfarin) was given to prevent clots.

CLI therapy is divided into conservative and operative management to improve symptoms and overcome the underlying disease that causes blood flow disorders. Conservative therapy consists of risk factors modification, including smoking cessation, control of blood pressure, blood glucose, and lipid profile (Hirsch et al., 2006). Peripheral arterial disease is a significant risk factor of morbidity and mortality in cardiovascular disease (Potier et al., 2011). Long-term modification therapy for risk factors modification is needed to reduce cardiovascular events. Low HDL level and high level of total and LDL cholesterol are associated with increased

cardiovascular mortality.(Hernando and Conejero, 2007) AHA recommends the usage of HMG CoA reductase inhibitors (statins) with LDL targets $<100 \mathrm{mg} / \mathrm{dl}$ and $<70 \mathrm{mg}$ / $\mathrm{dl}$ in patients at high risk of ischemia. Statins can reduce the risk of myocardial infarction and death from CHD by 24 to $34 \%$. Blood pressure control with antihypertensive drugs is needed with a target of less than 140/90 $\mathrm{mmHg}$ in nondiabetic patients, and less than $130 / 80 \mathrm{mmHg}$ in patients with diabetes and CKD (chronic kidney disease). Choice of antihypertensive drugs such as beta-adrenergic blockers and ACE inhibitors can be an option for reducing cardiovascular side effects (Hirsch et al., 2006).

The use of antiplatelets such as aspirin and clopidogrel is recommended to reduce the risk of myocardial infarction, stroke, and cardiovascular death in patients with PAD (Hirsch et al., 2006). A study shows that platelets play a role in the occurrence of atherosclerosis, where their activity is found to be increased by $30 \%$ higher in patients with peripheral vascular disease (Davies, 2012). Therefore, administration of antiplatelet agents such as aspirin has shown significant results in reducing risk and preventing reocclusion after revascularization therapy (Davies, 2012). The use of anticoagulants like warfarin can reduce the risk of cardiovascular events, but also increases the risk of bleeding events. Anticoagulants can be considered if other conditions, such as AF or prosthetic heart valves are found (Hirsch et al., 2006).

The administration of analgesic medication can be considered to reduce ischemic pain at rest and to overcome claudication complaints. Supervised exercise is also needed to help minimize claudication complaints. The use of vasodilators such as cilostazol has proven to be effective in improving symptoms and increasing walking distance in patients. Second-line therapy of cilostazol is pentoxifylline which is a derivate of methylxanthine which is able to reduce blood viscosity and inhibit adhesion and activation of neutrophils and fibrinogen. Prostaglandin derivatives such as beraprost and iloprost can exert vasodilatation effects and inhibit platelet aggregation (Hirsch et al., 2006).

Until now, there is no consensus with endovascular and conservative therapy selection, but the main goal of treatment is limb preservation.(Hirsch et al., 2006, Kinlay, 2016) If within 4-8 weeks there is no improvement from conservative therapy, or there is progression of symptoms or if wounds are becoming more widespread, the presence of disability and if the anatomy of the vascular system allows revascularization to be performed, then revascularization operative therapy must be carried out immediately, and amputation should also be performed if deemed necessary (Hirsch et al., 2006).

The patient carried out percutaneous revascularization transluminal angiography therapy to restore distal perfusion. The patient was given paracetamol to reduce pain and given cilostazol and beraprost sodium as vasodilators to improve claudication complaints. The administration of acetylsalicylate as antiplatelet and warfarin as anticoagulation in addition to being the therapy for AF is also given to reduce cardiovascular events and post PTA reocclusion. The patient also received atorvastatin as secondary prophylaxis.

\section{CONCLUSION}

A case of critical limb ischemia (CLI) has been reported in a diabetic patient with atrial fibrillation (AF). CLI is the final stage of peripheral arterial disease (PAD) with a high risk for limb loss and cardiovascular events. PAD in patients with diabetes is often under-recognized due to complications from peripheral neuropathy that disguises symptoms so that it is often found in its late stages. AF is the most common cause of embolism in peripheral blood vessels. $60-95 \%$ of patients who are operated on due to limb ischemia are also diagnosed with AF. Therapy of CLI is divided into conservative and operative management to improve symptoms and address underlying diseases that cause blood flow disorders. Early diagnosis and appropriate treatment can improve the prognosis and prevent limb loss.

\section{CONFLICT OF INTEREST}

The authors declare there is no conflict of interest.

\section{REFERENCES}

AHA. 2011. Management of patients with atrial fibrillation. Dallas: AHA. Beard JD. 2000. Chronic lower limb ischemia. BMJ, 320:854-857.

Bitar FG \& Garcia LA. 2010. Critical limb ischemia: an overview of the epidemiologic and clinical implications. Vascular Disease Management, 7(9).

Brandes A, Smit MD, Nguyen BO, Rienstra M, \& Gelder ICV. 2018. Risk factor management in atrial fibrillation. Arrhythmia \& Electrophysiology Review, 7(2):118-27.

Davies MG. 2012. Critical limb ischemia: epidemiology. MDCVJ, 8(4): 10-14.

Frost JL, Campos-Outcalt D, Hoelting D, LeFevre M, Lin KW, 
Vaughan W, Bird MD. 2017.

Pharmacologic management of newly detected atrial fibrillation. Leawood:AAFP.

Hernando FJS dan Conejero AM. 2007. Peripheral artery disease: pathophysiology, diagnosis and treatment. Rev Esp Cardiol, 60(9): 969-82.

Hirsch AT, Haskal ZJ, Hertzer NR, Bakal CW, Creager MA, Halperin JL, Hiratzka LF, Murphy WRC, Olin JW, Puschett JB, Rosenfield KA, Sacks D, Stanley JC, Taylor LM, White CJ, White J, White RA. 2006. ACC/ AHA 2005 Practice guidelines for the management of patients with peripheral arterial disease (lower extremity, renal, mesenteric, and abdominal aortic). Circulation, 113:e463-564.

Kinlay S. 2016. Management of critical limb ischemia. Circ Cardiovasc Interv, 9(2):e001946. Kuzniatsova N \& Lip GYH. 2011. Prevention of thromboembolism in atrial fibrillation patients.

European Cardiology, 7(1):37-43.

Li J, Luo Y, Xu Y, Yang J, Zheng L, Hasimu B, Yu J, \& Hu D. 2007. Risk factors of peripheral arterial disease and relationship between low ankle-brachial index and mortality form all- cause and cardiovascular disease in Chinese patients with type 2 diabetes. Circ J, 71: 377381 .

Menke J, Luthje L, Kastrup A, \& Larsen J. Thromboembolism in atrial fibrillation. The American Journal of Cardiology, 105(4):502-510.

Nehler MR, Duval S, Diao L, Annex BH, Hiatt WR, Rogers K, Zakharyan A, \& Hirsch AT. 2014. Epidemiology of peripheral arterial disease and critical limb ischemia in an insured national population. J Vasc Surg, 60(3):68695.

Papatheodorou K, Banach M, Bekiari E, Rizzo M, \& Edmonds M. 2018. Complications of Diabetes 2017. Journal of Diabetes Research, 2018:1-4.

Potier L, Abi Khalil C, Mohammedi K, \& Roussel R. 2011. Use and utility of ankle brachial index in patients with diabetes. Eur J Vasc Endovasc Surg, 41:110-116.

Spreen MI, Gremmels H, Teraa M, Sprengeres RW, Verhaar MC, Statius van Eps RG, PM de Vries JP, Mali WPTM, \& Hans van Overhagen. 2016. Diabetes is associated with decrease limb survival in patients with critical limb ischemia: pooled data from two randomized controlled trials. Diabetes Care, 39:2058-2064.

Thiruvoipati T, Kielhorn CE, \& Armstrong EJ. 2015. Peripheral artery disease in patients with diabetes: epidemiology, mechanisms, and outcomes. World Journal of Diabetes, 6(7): $961-969$.

Wasilewska M \& Gosk-Bierska I. 2013. Thromboembolism associated with atrial fibrillation as a cause of limb and organ ischemia. Adv Clin Exp Med, 22(6):865-873. 